\title{
Coding properties of spiking neurons: reverse and cross-correlations
}

\author{
Wulfram Gerstner \\ Swiss Federal Institute of Technology Lausanne, Laboratory of Computational Neuroscience, DI-LCN, CH 1015 Lausanne EPFL, Switzerland
}

Received 9 March 2001; accepted 9 March 2001

\begin{abstract}
What is the 'meaning' of a single spike? Spike-triggered averaging ('reverse correlations') yields the typical input just before a spike. Similarly, cross-correlations describe the probability of firing an output spike given (one additional) presynaptic input spike. In this paper, we analytically calculate reverse and cross-correlations for a spiking neuron model with escape noise. The influence of neuronal parameters (such as the membrane time constant, the noise level, and the mean firing rate) on the form of the correlation function is illustrated. The calculation is done in the framework of a population theory that is reviewed. The relation of the population activity equations to population density methods is discussed. Finally, we indicate the role of cross-correlations in spike-time dependent Hebbian plasticity. (C) 2001 Elsevier Science Ltd. All rights reserved.
\end{abstract}

Keywords: Spiking neurons; Integrate-and-fire model; Reverse correlations; Cross-correlations; Population equations; Population activity; Rate model; Hebbian learning; Spike-time dependent plasticity; Noise

\section{Introduction}

The problem of neural coding can be phrased as two questions (Berry \& Meister, 1998; Bialek, Rieke, de Ruyter van Steveninck \& Warland, 1991; de Ruyter van Steveninck, Lowen, Strong, Koberle \& Bialek, 1997; Kjaer, Hertz \& Richmond, 1994; König, Engel \& Singer, 1996; Optican \& Richmond, 1987; Rieke, Warland, de Ruyter van Steveninck \& Bialek, 1996; Roddey, Girish \& Miller, 2000; Softky, 1995; Tovee, Rolls, Treves \& Belles, 1993).

1. Encoding. What is the effect of a single presynaptic spike on the activity of a postsynaptic neuron, i.e. does the stimulation cause an output spike or not?

2. Decoding. What do we learn about the stimulus from the observation of a single output spike?

The first question can be addressed by cross-correlation experiments (see Fig. 1). A neuron that is spontaneously active because of background activity receives at time $t^{\text {in }}$ one additional presynaptic input spike. By averaging over many presynaptic spike arrivals at the same synapse, we can estimate the probability of generating an output spike $t^{(f)}$ given an input spike $t^{\text {in }}$. The cross-correlation $C^{\text {cross }}\left(t^{(f)} \mid t^{\text {in }}\right)$ can, therefore, be evaluated via a peri-stimulus-time histogram (PSTH) triggered on the presynaptic spike. Systematic experiments along those lines have, for example, been

E-mail address: wulfram.gerstner@epfl.ch (W. Gerstner). performed by Poliakov, Powers and Binder (1997) in continuation of earlier models and experiments (Fetz \& Gustafsson, 1983; Kirkwood \& Sears, 1978; Knox, 1974; Moore, Segundo, Perkel \& Levitan, 1970).

The second question can be addressed by information theoretic measurements (Bialek et al., 1991; de Ruyter van Steveninck et al., 1997; Optican \& Richmond, 1987; Tovee et al., 1993) or, in its simplest form by reverse-correlation measurements (de Boer \& Kuyper, 1968). In the reverse-correlation approach, a neuron is stimulated by a time-dependent stimulus $\mathscr{I}(t)=\mathscr{I}_{0}+\Delta \mathscr{I}(t)$. Every time an output spike $t^{(f)}$ occurs, the time course $\Delta \mathscr{I}\left(t^{(f)}-s\right)$ just before the firing time $t^{(f)}$ is recorded. Averaging over many firing times $t^{(f)}$ yields the typical input

$\left\langle\mathscr{I}\left(t^{(f)}-s\right)\right\rangle_{t^{(f)}}=C^{\mathrm{rev}}(s)$.

Thus, the reverse correlation function $C^{\mathrm{rev}}(s)$ is defined by a spike-triggered average (Fig. 2).

For a linear rate model where output spikes $t^{(f)}$ are generated by an inhomogeneous Poisson process with inputdependent rate

$v^{\text {out }}(t)=v_{0}^{\text {out }}+\int_{0}^{\infty} G(s) \Delta \mathscr{I}(t-s) \mathrm{d} s$

it is well known (de Boer \& Kuyper, 1968) that the reverse correlation function is determined by the filter $G(s)$ and the auto-correlation $\left\langle\Delta \mathscr{I}(t) \Delta \mathscr{I}\left(t^{\prime}\right)\right\rangle$ of the input (see Appendix A for a review of the argument). 
a)

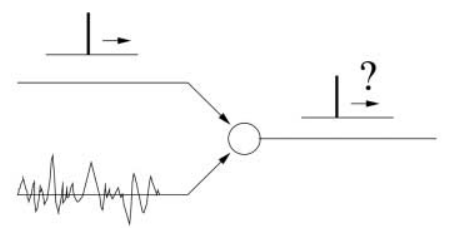

b)

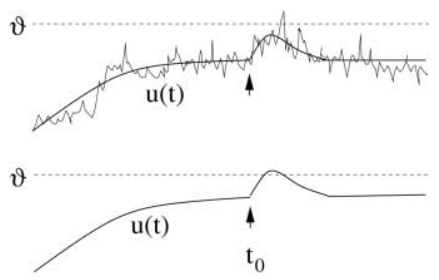

Fig. 1. (a) A neuron which is driven by a noisy background input receives at time $t_{0}$ one extra input spike. Does this extra input trigger an output spike? (b) Two hypothetic scenarios: top: with noisy input an output spike is the more likely the closer the mean membrane potential (thick solid line) is to the threshold $\vartheta$ (dashed horizontal line). The firing probability increases during the postsynaptic potential that is caused by the input pulse at $t_{0}$ (arrow); bottom: without noise, the membrane potential can reach threshold (for the first time) only during the rising phase of the postsynaptic potential.

In particular, for white-noise input with

$$
\langle\Delta \mathscr{I}(t)\rangle=0 \text { and }\left\langle\Delta \mathscr{I}(t) \Delta \mathscr{I}\left(t^{\prime}\right)\right\rangle=\sigma^{2} \delta\left(t-t^{\prime}\right)
$$

we have

$C^{\mathrm{rev}}(s)=\frac{\sigma^{2}}{v_{0}^{\text {out }}} G(s)$.

For spiking neurons, however, the form of the reverse correlation function and its relation to elementary model parameters such as the membrane time constant has remained unclear. Apart from occasional simulation results (e.g. Softky, 1995), no systematic study seems to have been undertaken. Oddly enough, it is recent progress in the theory of population dynamics (Abbott \& van Vreeswijk, 1993; Amit \& Brunel, 1997; Brunel \& Hakim, 1999; Fusi \& Matteo, 1999; Gerstner, 1995, 2000; Gerstner \& van Hemmen, 1992; Knight, 1972a; Nykamp \& Tranchina, 2000; Omurtag, Knight \& Sirovich, 2000) that allows us now to calculate some of the coding properties of single neurons (Bethge, Pawelzik, Rothenstein \& Tsodyks, 2001; Brunel, Chance, Fourcaud \& Abbott, 2001; Herrmann \& Gerstner, 1999, 2001a; see also Knight, 1972b). In fact, the typical behavior of a single neuron is identical to that of a population of independent neurons (i.e. without coupling).

The paper is organized as follows. After a brief discussion of the dynamics of a single spiking neuron in Section 2, we will review in Section 3 population equations that describe the dynamics of a pool of neurons. Linearization of the population equations allows us to analytically calculate the reverse correlation function for the case of input with

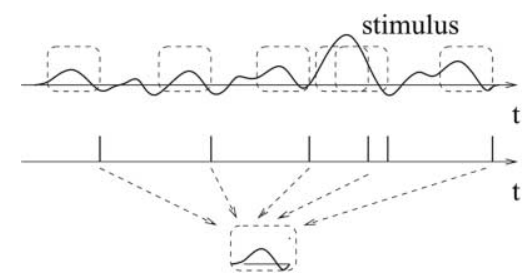

Fig. 2. Reverse correlation technique (schematic). The stimulus $\mathscr{I}(t)$ in the top trace has caused the spike train shown immediately below. The time course of the stimulus just before the spikes (dashed boxes) has been averaged to yield the typical time course $\left\langle\mathscr{I}\left(t^{(f)}-s\right)\right\rangle_{t^{(f)}}$ (bottom). low-amplitude fluctuations $\Delta \mathscr{I}(t)$. Reverse and cross-correlations are the topic of Section 4. In the discussion of Section 5 we indicate how the correlation function enters into the formalism of spike-time dependent Hebbian learning. Formal mathematical arguments have been moved to Appendices A and B.

\section{Spike response model}

In this section, we review the spike response model (SRM) and specify the particular, somewhat simplified version that we will use throughout the paper.

\subsection{General framework}

During and immediately after an action potential, the membrane potential of a given neuron follows (at least in most cases) a rather stereotyped trajectory. In the SRM, this trajectory is described as a function $\eta(t-\hat{t})$ where $\hat{t}$ is the moment when the spike has been triggered. The form of the function $\eta$ can be chosen so as to reproduce as closely as possible the time course of an action potential. If a short current pulse $\mathscr{I}(t)$ is applied during or immediately after an action potential, the membrane potential $u(t)$ will deviate slightly from its standard time course $\eta(t-\hat{t})$. The difference between the actual time course $u(t)$ and the reference time course $\eta(t-\hat{t})$ is described by a response kernel $\kappa$, i.e.

$u(t)=\eta(t-\hat{t})+\int_{0}^{\infty} \kappa(t-\hat{t}, s) \mathscr{I}(t-s) \mathrm{d} s$.

Note that the response kernel $\kappa$ depends on the time $t-\hat{t}$ since the last spike. In order to emphasize this dependence, we introduce a refractory variable $r=t-\hat{t}$ and rewrite Eq. (5) in the form

$u_{r}(t)=\eta(r)+\int_{0}^{\infty} \kappa(r, s) \mathscr{I}(t-s) \mathrm{d} s$

If the last spike occurred a long time back in the past and if there has been no further stimulation, the neuron is at rest. In this case, the response to a small current pulse is described by $\kappa_{0}(s)=\lim _{r \rightarrow \infty} \kappa(r, s)$; typically the time course $\kappa_{0}(s)$ is characterized by the passive membrane time constant $\tau_{\mathrm{m}}$. During or a few milliseconds after an action potential, the 
a)

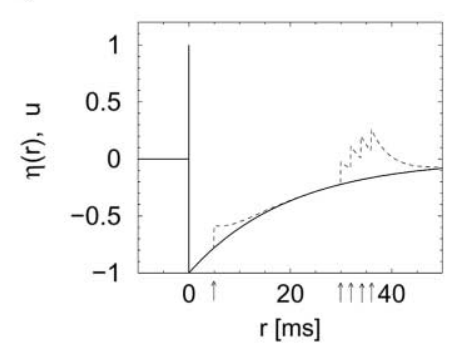

b)

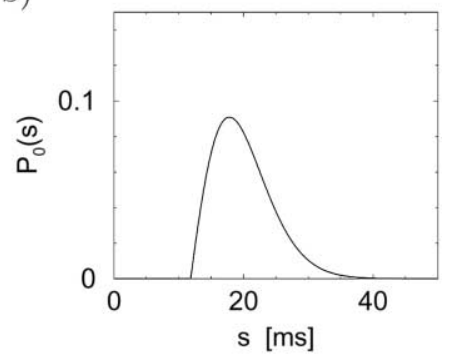

Fig. 3. (a) Spike Response Model. A neuron that has fired at $r=0$ (vertical line) passes for $r>0$ through a phase of refractoriness described by a kernel $\eta(r)=-\eta_{0} \exp \left(-r / \tau_{\text {refr }}\right)$ with $\tau_{\text {refr }}=20 \mathrm{~ms}$ (solid line). Each presynaptic current pulse (arrow) causes a response $\kappa(r, s) \propto \exp \left(-s / \tau_{\mathrm{m}}\right)$ with $\tau_{\mathrm{m}}=4 \mathrm{~ms}$. The membrane potential $u$ is the sum of the refractory kernel and the total effect of input current (dashed line). (b) Inter-spike interval distribution $P_{0}(s)$ for the piecewise linear escape rate (13) with $\beta=1$. The neuron is stimulated by a constant current $\mathscr{I}_{0}$ that has been adjusted so that the mean interval is $\langle s\rangle=\int_{0}^{\infty} s P_{0}(s) \mathrm{d} s=20 \mathrm{~ms}$.

response to the same current pulse can be quite different since sodium and potassium channels are open that are normally closed at rest. The dependence of $\kappa$ upon the refractory variable $r$ takes care of this effect (Kistler, Gerstner \& van Hemman, 1997).

In a noiseless model neuron, spikes are generated by a threshold process that resets the refractory variable $r$ in Eq. (6)

$$
u(t)=\vartheta \Rightarrow r=0 \quad(\hat{t}=t) .
$$

Graphically speaking, we simply add (or 'paste in') an action potential of standard shape $\eta(t-\hat{t})$ if the membrane potential reaches the threshold at time $\hat{t}$. Noise can be induced by adding a stochastic component to the input current $\mathscr{I}(t) \rightarrow \mathscr{I}(t)+\mathscr{I}_{\text {noise }}(t)$. The stochastic contribution $\mathscr{I}_{\text {noise }}$ can be motivated by stochastic spike arrival. Usually the noise is taken as white, i.e. $\left\langle\mathscr{I}_{\text {noise }}\right\rangle=0$ and $\left\langle\mathscr{I}_{\text {noise }}(t) \mathscr{I}_{\text {noise }}\left(t^{\prime}\right)\right\rangle=\sigma_{n}^{2} \delta\left(t-t^{\prime}\right)$. We call this the diffusive noise model. The noisy input generates a distribution of membrane potentials with standard deviation $\sigma_{u}$ that can be measured in experiments (Destexhe \& Pare, 1999). While such a noise model is not too far from reality, it suffers from the disadvantage that it renders the analytical calculation of inter-spike interval distributions rather difficult.

It is, therefore, more convenient (albeit somewhat less realistic) to introduce noise by replacing the deterministic threshold by a 'noisy' threshold. More precisely, the neuron may fire in a time step $\Delta t$ with some probability $\rho \Delta t$ even though the membrane potential $u$ has not yet reached the threshold $\vartheta$. The 'hazard' $\rho$ of firing depends on the momentary distance between the membrane potential and the threshold and, possibly, also on the slope $\dot{u}=\mathrm{d} u / \mathrm{d} t$, i.e. ${ }^{1}$

$\rho(t)=f[u(t)-\vartheta, \dot{u}]$.

We call $f$ the 'escape rate'. A suitable function $f$ should vanish for $u \ll \vartheta$ and increase rapidly for $u \gg \vartheta$. It turns

\footnotetext{
${ }^{1}$ In addition, we require that the rate vanishes during the first few milliseconds that correspond to the duration of the spike itself.
}

out that, at least for standard integrate-and-fire neurons, the diffusive noise model introduced above can be replaced to a high degree of accuracy by an escape rate of the form (Herrmann \& Gerstner, 2001a; Plesser \& Gerstner, 2000;)

$f(u=\vartheta, \dot{u})=a\left(\frac{1}{\tau}+2 \dot{u}\right) \frac{\mathscr{G}\left(u-\vartheta, \sigma_{u}\right)}{\operatorname{Erfc}\left(\frac{u-\vartheta}{\sigma_{u}}\right)}$

where $\mathscr{G}\left(x, \sigma_{u}\right)$ is a normalized Gaussian with standard deviation $\sigma_{u}$ and vanishing mean; $\operatorname{Erfc}(x)$ is the complementary error function; and $a$ is a parameter. Since in an integrate-and-fire model, the variance $\sigma_{u}^{2}$ of the membrane potential is directly related to the (usually known) variance $\sigma_{n}^{2}$ of the noise current, $a$ is in fact the only free parameter for a fit of the escape rate (9) to the diffusive noise model. Optimization of $a$ over a large ensemble of periodic and aperiodic stimuli yields $a^{\mathrm{opt}} \approx 1.2$ (H.E. Plesser, private communication). Note that for $u \gg \vartheta$ and fixed $\dot{u}$, the escape rate (9) increases linearly with $u-\vartheta$.

A major advantage of escape rate models is the fact that statistical quantities such as the interval distribution can be calculated analytically. In fact, whatever the choice of the escape rate $f$, the probability that a neuron fires a spike at time $t$ given that its last spike was at $\hat{t}$ is

$P_{\mathscr{A}}(t \mid \hat{t})=\rho_{r}(t) \exp \left\{-\int_{\hat{t}}^{t} \rho_{r}\left(t^{\prime}\right) \mathrm{d} t^{\prime}\right\}$

where $\rho_{r}\left(t^{\prime}\right)=f\left[u_{r}\left(t^{\prime}\right)-\vartheta, \dot{u}_{r}\left(t^{\prime}\right)\right]$ with $r=t^{\prime}-\hat{t}$; cf. Eq. (6). For constant input $\mathscr{I}(t)=\mathscr{I}_{0}$, we get the stationary interval distribution $P_{0}(t-\hat{t}) \equiv P_{\mathscr{I}_{0}}(t \mid \hat{t})$. For arbitrary time-dependent input, we call $P_{\mathscr{A}}(t \mid \hat{t})$ the input-dependent interval distribution. Eq. (10) is a generalization of standard renewal theory (Cox, 1962; Perkel, Gerstein \& Moore, 1967) to the case of time-dependent input.

\subsection{Specific model}

In this paper, we work in the framework of the noisy SRM defined by Eqs. (6) and (8). It has been shown that with an optimal choice of the kernels $\eta$ and $\kappa$ the SRM can 
correctly predict about $90 \%$ of the firing times of the Hodgkin-Huxley model with time-dependent input (Kistler et al., 1997). Thus, the SRM seems to offer a framework that is general enough to capture some essential aspects of neuronal dynamics.

To keep our arguments transparent, we focus in this paper on a much simpler model within the framework of Eq. (6) and take exponential kernels

$$
\begin{aligned}
& \eta(r)=\delta(r)-\eta_{0} \exp \left(-\frac{r}{\tau_{\text {refr }}}\right) \mathscr{H}(r) \\
& \kappa(r, s)=\exp \left(-\frac{s}{\tau_{\mathrm{m}}}\right) \mathscr{H}(r-s) \mathscr{H}(s)
\end{aligned}
$$

where $\mathscr{H}($.$) is the Heaviside step function; cf. Fig. 3a. In Eq.$ (11), the form of an action potential has been reduced to a Dirac $\delta$ function which simply marks the firing time of the spike. The Heaviside function $\mathscr{H}(r-s)$ in Eq. (12) assures that the membrane potential trajectory restarts after each spike with an initial condition $\lim _{r \rightarrow 0} u_{r}(t)=-\eta_{0}$. Our specific choice of parameters is $\tau_{\mathrm{m}}=4 \mathrm{~ms}, \tau_{\text {refr }}=20 \mathrm{~ms}$, and $\eta_{0}=1$; cf. Fig. 3a.

For the escape rate, we take the piecewise linear function

$\rho_{r}(t)=\beta\left[u_{r}(t)-\vartheta\right]_{+}=\beta\left[u_{r}(t)-\vartheta\right] \mathscr{H}\left[u_{r}(t)-\vartheta\right]$.

A value of $\beta=1$ corresponds to a medium noise level; $\mathrm{cf}$. Fig. 3b.

We note that for $\tau_{\text {refr }}=\tau_{\mathrm{m}} \equiv \tau$ we obtain the standard integrate-and-fire neuron as a special case. In fact, taking the derivative of Eq. (5) with exponential kernels $\eta$ and $\kappa$ with a common time constant $\tau$ yields between two spikes

$$
\frac{\mathrm{d}}{\mathrm{d} t} u=-\frac{u}{\tau}+\mathscr{I}(t)
$$

After firing, the membrane potential is reset and integration restarts at a value $u_{\text {reset }}=-\eta_{0}$. For $\beta \rightarrow \infty$ firing occurs immediately when $u(t)$ crosses the threshold. In other words, in the limit of $\beta \rightarrow \infty$ and $\tau_{\text {refr }}=\tau_{\mathrm{m}}$ we recover the noise-free integrate-and-fire neuron. On the other hand, a choice $\tau_{\text {refr }} \neq \tau_{\mathrm{m}}$ allows us to separate the time scale of refractoriness from the time scale set by the membrane time constant which is not possible in the case of a standard integrate-and-fire model.

\section{Population equations}

Instead of studying a single neuron and averaging over a long experiment (or several trials) to measure the reverse correlations, we will consider in this paper a population of $N$ independent neurons and study its behavior in a single (short) trial. Let us denote the spike train of neuron $j$ by $S_{j}(t)=\sum_{f} \delta\left(t-t_{j}^{(f)}\right)$ where $t_{j}^{(f)}$ with $f=1,2, \ldots$ are the firing times of neuron $j$. The quantity we are interested in is the population activity

$A(t)=\lim _{\Delta t \rightarrow 0} \frac{1}{\Delta t N} \sum_{j=1}^{N} \int_{t}^{t+\Delta t} S_{j}\left(t^{\prime}\right) \mathrm{d} t^{\prime}=\frac{1}{N} \sum_{j=1}^{N} \sum_{f} \delta\left(t-t_{j}^{(f)}\right)$,

i.e. the number of spikes that occur in a population of $N$ neurons in a time window $[t, t+\Delta t]$ divided by the number of neurons and the length of the window. Since the population activity has units of a rate, it is also called the population rate (Nykamp \& Tranchina, 2000) or the space-rate (Maas \& Nathschläger, 2000).

In order to get a first intuitive understanding of the relation between the population activity and single-neuron dynamics, let us consider a population which is stimulated at $t=0$ by a stimulating pulse $\mathscr{I}(t)$ of a duration of, say, $2 \mathrm{~ms}$. Neurons in the population that happen to be close to threshold at the moment of input switching will be put across the threshold by the stimulation and will, therefore, emit an action potential. Other neurons that are in the refractory period or close to rest will stay quiescent. Thus, on a single-neuron level the response to a stimulus can be completely different depending on the internal state of the neuron. By calculating the population activity $A(t)$, we average over the internal state - in very much the same way as a repetition of the stimulus at random intervals would do.

In this section, we review the population dynamics of spiking neurons and derive a population equation of the form (Gerstner, 1995, 2000; Gerstner \& van Hemmen, 1992)

$A(t)=\int_{-\infty}^{t} P_{\mathscr{I}}(t \mid \hat{t}) A(\hat{t}) \mathrm{d} \hat{t}$

where $P_{\mathscr{I}}(t \mid \hat{t})$ is the input-dependent interval distribution introduced in Eq. (10). In particular, we discuss the relation of this integral equation to the membrane potential density approach of Abbott and van Vreeswijk (1993); Nykamp and Tranchina (2000); Omurtag et al. (2000) and Brunel and Hakim (1999).

\subsection{Review of density methods}

Before we turn to the SRM, we make a short detour and start with the well-known integrate-and-fire model; cf. Eq. (14). Since the state of individual integrate-and-fire neurons is fully characterized by their membrane potential, the state of a large population of integrate-and-fire neurons can be described by a membrane potential density $p(u, t)$. The normalization is $\int_{-\infty}^{\vartheta} p(u, t) \mathrm{d} u=1$, i.e. $\int_{u_{0}}^{u_{1}} p(u, t) \mathrm{d} u$ is the probability that a randomly chosen neuron has at time $t$ a membrane potential between $u_{0}$ and $u_{1}$. The dynamics of the membrane potential density can be expressed by the continuity equation (Abbott \& van Vreeswijk, 1993; Brunel \& Hakim, 1999; Nykamp \& 
a)

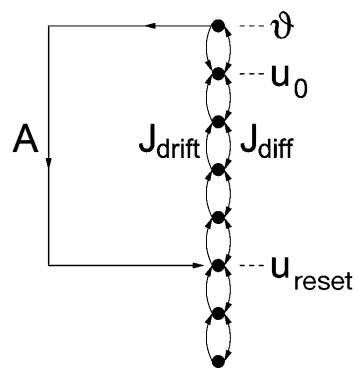

b)

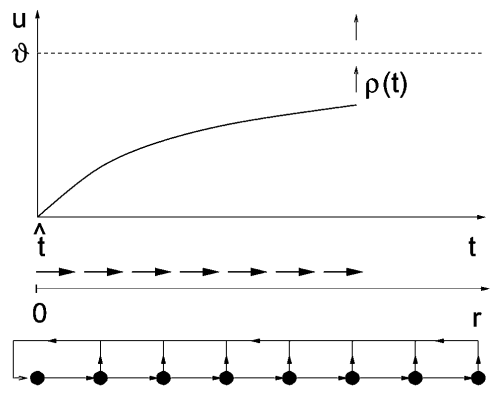

Fig. 4. (a) State transitions in a discretized implementation of the membrane potential density equation for integrate-and-fire neurons stimulated by constant current $\mathscr{I}_{0}$ and diffusive noise. The flux $J_{\text {drift }}$ drives the membrane potential towards $u_{0}=R \mathscr{I}_{0}$, while stochastic spike arrival (diffusive noise) leads to a flux $J_{\text {diff }}$ upwards (excitation) or downwards (inhibition). The flux across threshold, i.e. the activity $A$, is reinjected at the reset potential $u_{\text {reset }}$ (schematic). (b) Population dynamics of SRM neurons with escape noise. Top: membrane potential of a neuron that has fired its last spike at $\hat{t}$. The membrane potential trajectory $u(t)$ ends when the neuron fires. The instantaneous firing rate is $\rho_{r}(t)=f\left[u_{r}(t)-\vartheta\right]$. Middle: the flow has constant speed along the $r$-axis. The trajectory of the refractory variable $r(t)$ ends when the neuron fires and restarts immediately at $r=0$. Bottom: discretized state diagram; the activity $A$ consists of the firings of all neurons with different refractory variables $r$. The activity $A$ is reinjected at $r=0$.

Tranchina, 2000; Omurtag et al., 2000)

$$
\frac{\partial}{\partial t} p(u, t)=-\frac{\partial}{\partial u} J(u, t)+A(t) \delta\left(u-u_{\text {reset }}\right)
$$

where $J(u, t)$ is the flux along the voltage coordinate and $A(t)$ is the population activity. The last term in Eq. (17) describes the reset to $u=u_{r}$ after firing. For neurons with stochastic input $\mathscr{I}_{\text {noise }}$ of power $\sigma_{n}^{2}$, the flux is

$J(u, t)=p(u, t) \dot{u}-\frac{\sigma_{n}^{2}}{2 \tau} \frac{\partial}{\partial u} p(u, t)$

where $\dot{u}=(-u / \tau)+\mathscr{I}(t)$ is the velocity of the flow. The second term in Eq. (18) arises due to the noise component $\mathscr{I}_{\text {noise }}$ and is called the 'diffusion term'. The population activity $A(t)$ is given by the flux through the threshold; cf. Fig. 4a. Since the threshold acts as an absorbing boundary (i.e. $p(u, t)=0$ for $u \geq \vartheta$ ), we have

$A(t)=J(\vartheta, t)=\frac{\sigma_{n}^{2}}{2 \tau} \frac{\partial}{\partial u} p(\vartheta, t)$.

For stationary input, Eqs. (17)-(19) can be solved analytically (Brunel \& Hakim, 1999; Riccardi, 1977); for arbitrary timedependent input they must be integrated numerically (Nykamp \& Tranchina, 2000; Omurtag et al., 2000). This ends our discussion of integrate-and-fire neurons with diffusive noise.

We now return to neurons of the SRM type. The interval state of a SRM-neuron can be characterized by the time $r=$ $t-\hat{t}$ since its last output spike. We, therefore, introduce a 'refractory density' $q(r, t)$ with normalization

$\int_{0}^{\infty} q(r, t) \mathrm{d} r=1$,

i.e. $\int_{r_{0}}^{r_{1}} q(r, t) \mathrm{d} r$ is the probability that a randomly chosen neuron has at time $t$ a refractory variable between $r_{0}$ and $r_{1}$. In analogy to Eq. (17), the continuity equation for SRM neurons is given by Gerstner and van Hemmen (1992)

$\frac{\partial}{\partial t} q(r, t)=-\frac{\partial}{\partial r} J(r, t)-\rho_{r}(t) q(r, t)+A(t) \delta(r)$.
The flux along the $r$-axis is

$J(r, t)=q(r, t) \dot{r}=q(r, t)$

since the velocity $\dot{r}$ of the refractory variable is constant and equal to one. The last term in Eq. (21) describes the 'reset' of the refractory variable to $r=0$. Note that, in contrast to diffusive noise, escape noise does not enter into the flux equation (22) but directly as a 'sink' term $-\rho_{r}(t) q(r, t)$ on the right-hand-side of Eq. (21). The magnitude of the 'sink' can be calculated from the escape rate

$\rho_{r}(t)=f\left[u_{r}(t)-\vartheta, \dot{u}_{r}(t)\right]$

with $u_{r}$ given by Eq. (6). The population activity is

$A(t)=\int_{0}^{\infty} \rho_{r}(t) q(r, t) \mathrm{d} r$,

i.e. neurons with refractory variable $r$ contribute with rate $\rho_{r}(t)$ to the activity $A(t)$. A schematic graph of the refractory dynamics is given in Fig. $4 \mathrm{~b}$.

We show in Appendix B that, for integrate-and-fire neurons with escape noise (and similarly for noiseless neurons), the continuity equation (17) for the membrane potential density $p(u, t)$ is equivalent to the continuity equation (21) for the refractory density $q(r, t)$. In fact, the transition from Eq. (17) to Eq. (21) is given by a simple coordinate transformation. The advantage of the formulation with refractory densities is that the partial differential equation (21) can be solved analytically. Since the flux has the simple form (Eq. (22)), we may switch to a moving coordinate frame. In the new frame, the partial differential equation has been reduced to an ordinary differential equation that can be integrated; cf. Appendix B. The result is (Gerstner \& van Hemmen, 1992, 1994)

$A(t)=\int_{-\infty}^{t} \rho_{t-\hat{t}}(t) \exp \left[-\int_{\hat{t}}^{t} \rho_{t^{\prime}-\hat{t}}\left(t^{\prime}\right) \mathrm{d} t^{\prime}\right] A(\hat{t}) \mathrm{d} \hat{t}$,

which has the form of the population equation (16) with an input-dependent interval distribution given by Eq. (10). The 
correct normalization of the population activity $A$ follows from Eq. (20) and is given by

$$
1=\int_{-\infty}^{t} \exp \left[-\int_{\hat{t}}^{t} \rho_{t^{\prime}-\hat{t}}\left(t^{\prime}\right) \mathrm{d} t^{\prime}\right] A(\hat{t}) \mathrm{d} \hat{t} .
$$

\subsection{Linearization}

In this subsection, we linearize the population equation (16) about a constant activity level $A_{0}$. The activity $A_{0}$ can be the result of stimulation by a constant current $\mathscr{I}_{0}>0$ or else due to spontaneous activity of the neurons $\left(\mathscr{I}_{0}=0\right)$. Let us now assume that, in addition to the bias current $\mathscr{I}_{0}$, we also apply a time-dependent input $\Delta \mathscr{I}(t)$. The population activity will then respond by a change $\Delta A(t)$, i.e.

$A(t)=A_{0}+\Delta A(t)$.

Linearization of the population equation (16) yields (Gerstner, 2000)

$\Delta A(t)=\int_{-\infty}^{t} P_{0}(t-\hat{t}) \Delta A(\hat{t})+A_{0} \frac{\mathrm{d}}{\mathrm{d} t} \int_{0}^{\infty} \mathscr{L}(x) \int_{0}^{\infty} \kappa_{0}(s) \Delta \mathscr{I}(t-x-s) \mathrm{d} s \mathrm{~d} x$.

The first term on the right-hand-side of Eq. (28) describes the after-effect of previous perturbations $\Delta A(\hat{t})$ in the past $(\hat{t}<t)$. Perturbations that have occurred about one interspike interval earlier 'reappear' at time $t$, smoothed out by the interval distribution $P_{0}(\hat{t})$. If there have been no perturbations in the past, the first term vanishes. The second term in Eq. (28) is a linear filter that describes the immediate effect of recent input variations $\Delta \mathscr{I}\left(t^{\prime}\right)$.

Three aspects are important for an understanding of the filter properties generated by the second term in Eq. (28). Firstly, the integral $\Delta h(t)=\int_{0}^{\infty} \kappa_{0}(s) \Delta \mathscr{I}(t-s) \mathrm{d} s$ can be interpreted as the contribution to the membrane potential caused by the input $\Delta \mathscr{I}$. We call $\Delta h$ the input potential.

Secondly, the input potential is convolved with a filter $\mathscr{L}(x)$. For escape rates $f(u)$ (i.e. escape rates that depend on the voltage $u$, but not on the slope $\dot{u}$ ), the filter is (Gerstner, 2000)

$\mathscr{L}(x)=\int_{x}^{\infty} f^{\prime}[u(\xi-x)-\vartheta] S_{0}(\xi) \mathrm{d} \xi-S_{0}(x) \int_{0}^{x} \mathrm{e}^{-\xi / \tau} f^{\prime}[u(\xi)-\vartheta] \mathrm{d} \xi$,

with

$S_{0}(s)=\exp \left\{-\int_{0}^{s} f[u(\xi)-\vartheta] \mathrm{d} \xi\right\}$.

Here, $u(\xi)$ is the membrane potential trajectory of a neuron with constant stimulus $\mathscr{I}_{0}$. As we can see from Fig. 5, for high noise the filter $\mathscr{L}(x)$ exhibits low-pass characteristics (i.e. the filter extends over a broad time window of averaging), whereas for low noise it becomes sharper. In the limit of no noise $(\beta \rightarrow \infty)$, it approaches a $\delta$ function.

Thirdly, Eq. (28) combines the integral over the filter $\mathscr{L}$ with a temporal derivative. For high noise, the derivative 'cancels' the convolution by the low-pass filter $\mathscr{L}$. The

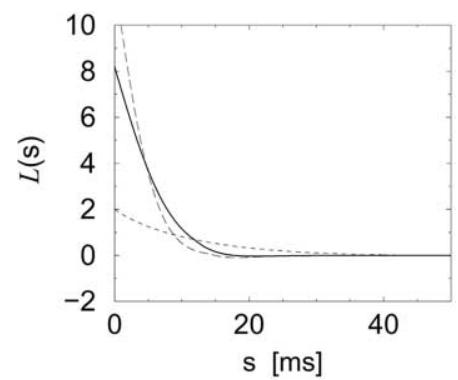

Fig. 5. Filter $\mathscr{L}(s)$ for a SRM neuron with exponential kernels and a piecewise linear escape rate (13) at a noise level of $\beta=1$ (solid line), $\beta=0.1$ (dotted line), and $\beta=2$ (long-dashed line). The bias current $\mathscr{I}_{0}$ has been readjusted so that the mean interval is always $20 \mathrm{~ms}$ (mean firing rate $v_{0}^{\text {out }}=50 \mathrm{~Hz}$ ). Parameters: membrane time constant $\tau_{\mathrm{m}}=4 \mathrm{~ms}$ and refractory time constant $\tau_{\text {refr }}=20 \mathrm{~ms}$.

immediate response $\Delta A$ to a change in the input is, therefore, proportional to the input potential

high noise : $\Delta A(t) \propto \Delta h(t)=\int_{0}^{\infty} \kappa(s) \Delta \mathscr{I}(t-s) \mathrm{d} s$.

For low noise, however, the immediate response becomes proportional to the derivative of the input potential

low noise : $\Delta A(t) \propto \frac{\mathrm{d}}{\mathrm{d} t} \Delta h(t)=\frac{\mathrm{d}}{\mathrm{d} t} \int_{0}^{\infty} \kappa(s) \Delta \mathscr{I}(t-s) \mathrm{d} s$.

An advantage of the analytical expressions (28) and (29) is that we can not only study the extreme cases but also intermediate noise levels. An illustration is given in the following section, where we determine reverse and crosscorrelation functions.

\section{Results}

In this section, we apply the linearized population equation to the problem of neuronal coding and calculate reverse and cross-correlation functions. The Fourier transform of Eq. (28) yields

$\hat{A}(\omega)=i \omega \frac{A_{0} \hat{\mathscr{L}}(\omega) \hat{\kappa}(\omega)}{1-\hat{P}(\omega)} \hat{\mathscr{I}}(\omega)=\hat{G}(\omega) \hat{\mathscr{I}}(\omega)$

with a filter

$\hat{G}(\omega)=i \omega \frac{A_{0} \hat{\mathscr{L}}(\omega) \hat{\kappa}(\omega)}{1-\hat{P}(\omega)}$

The inverse Fourier transform of Eq. (33) yields

$A(t)=A_{0}+\int_{0}^{\infty} G(s) \Delta \mathscr{I}(t-s) \mathrm{d} s$

where $A_{0}$ is the population activity for stimulation with constant bias current $\mathscr{I}_{0}$. The filter $G$ is

$G(s)=\frac{1}{2 \pi} \int_{0}^{\infty} \hat{G}(\omega) \mathrm{e}^{+i \omega s} \mathrm{~d} \omega$. 
a)

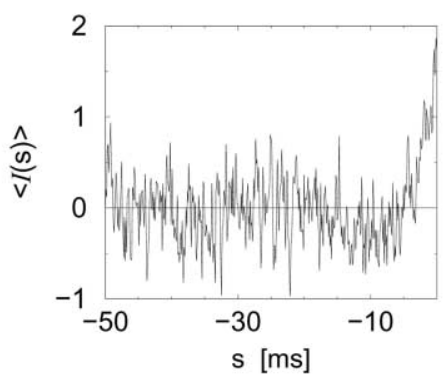

b)

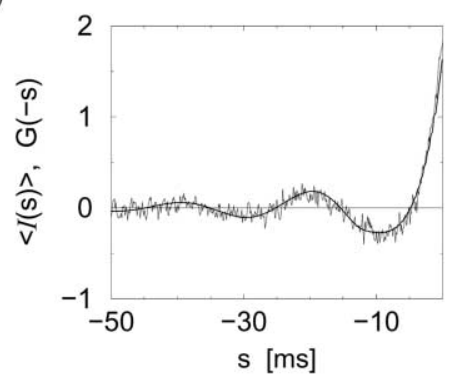

Fig. 6. Reverse correlations. A SRM $\mathrm{SM}_{0}$ neuron is stimulated by a constant bias current plus a stochastic input current. Each time an output spike is triggered, the time course of the input current is recorded. (a) Average input $\left\langle\mathscr{I}\left(t-t^{(f)}\right)\right\rangle_{t^{(f)}}$ as a function of $s=t-t^{(f)}$ averaged over 1000 output spikes $f=1, \ldots, 1000$. (b) The same, but averaged over 25,000 spikes. The simulation result is compared with the time-reversed linear filter $G(-s)$ predicted from the theory (smooth line). Noise model: linear escape rate with $\beta=1$.

Eq. (35) has the form of a linear rate model; cf. Eq. (2). We can, therefore, repeat the arguments of Appendix A to show that

$C^{\mathrm{rev}}(s)=\frac{\sigma^{2}}{A_{0}} G(s)$

The central point of our approach is that the filter $G(s)$ can be predicted via Eq. (34) from the shape of the interval distribution $P_{0}(s)$, the response kernel $\kappa_{0}(s)$ and the filter $\mathscr{L}(s)$. Eqs. (34) and (37) establish, therefore, a relation between neuronal parameters and the reverse correlation function $C^{\mathrm{rev}}(s)$.

\subsection{Reverse correlations}

We consider the SRM neuron defined in Section 2.2. The response kernels are exponential kernels with a time constant of $\tau_{\mathrm{m}}=4 \mathrm{~ms}$ for the kernel $\kappa$ and $\tau_{\text {refr }}=20 \mathrm{~ms}$ for the refractory kernel $\eta$. The neuron is driven by a current $\mathscr{I}(t)=\mathscr{I}_{0}+\Delta \mathscr{I}(t)$. The bias current $\mathscr{I}_{0}$ was adjusted so that the neuron fired at a mean rate of $50 \mathrm{~Hz}$. The noise current was generated by the following procedure. In every time step of $0.1 \mathrm{~ms}$ we apply with a probability of 0.5 an input pulse of amplitude \pm 1 , i.e. the input has whitenoise characteristics. To estimate the reverse correlation function, we build up a histogram of the average input $\left\langle\mathscr{I}\left(t-t^{(f)}\right)\right\rangle_{t^{(f)}}$ preceding a spike $t^{(f)}$. We see from Fig. 6a that the main characteristics of the reverse correlation func-

a)

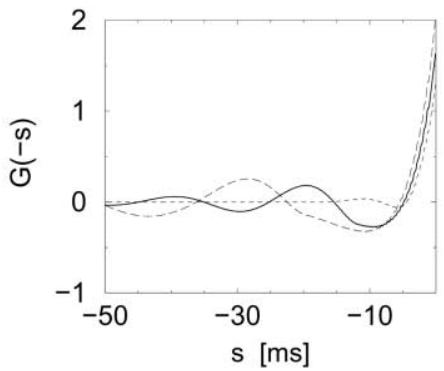

tion are already visible after 1000 spikes. After an average over 25,000 spikes, the time course is much cleaner and reproduces to a high degree of accuracy the time course of the time-reversed filter $G(-s)$ predicted by the theory. The oscillation with a period of about $20 \mathrm{~ms}$ reflects the intrinsic firing period of the neuron. This is shown more clearly in Fig. 7 where the bias current has been changed so that the mean interval is either shorter $(\langle s\rangle=10 \mathrm{~ms})$ or longer $(\langle s\rangle=30 \mathrm{~ms})$. The oscillations of the reverse correlation filter $G(-s)$ change accordingly. An increase in the noise level suppresses the oscillations; cf. Fig. 8.

\subsection{Cross-correlations}

In the preceding subsection, the input $\Delta \mathscr{I}(t)$ was a random current with white-noise characteristics; cf. Eq. (3). In this subsection, we focus on a scenario that is somewhat closer to real synaptic input. To keep things simple, we model presynaptic spike arrival as current injection (rather than conductance change). More precisely, we assume that a presynaptic spike that arrives at time $t^{\text {in }}$ generates an exponential current pulse

$\mathscr{I}^{\mathrm{syn}}(t)=\frac{q}{\tau_{\mathrm{syn}}} \exp \left(-\frac{t-t^{\text {in }}}{\tau_{\mathrm{syn}}}\right) \mathscr{H}\left(t-t^{\text {in }}\right)$

where $\mathscr{H}($.$) is the Heaviside step function. The postsynaptic$

b)

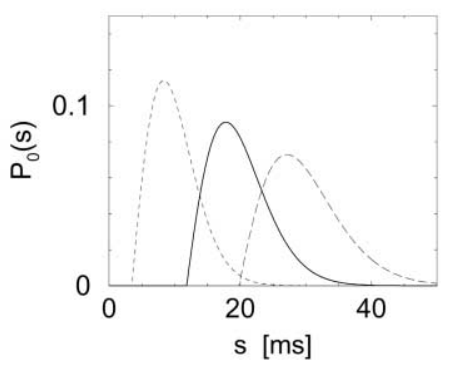

Fig. 7. Reverse correlations (a) and interval distribution (b) for the same neuron as in Fig. 6, except that the bias current has been changed so that the mean interval is 10 (dotted line), 20 (solid line) or $30 \mathrm{~ms}$ (long-dashed line). Linear escape rate with $\beta=1$. 
a)

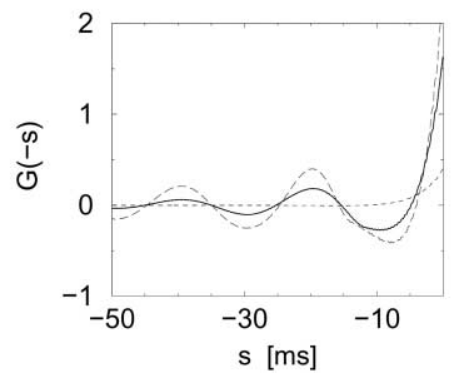

b)

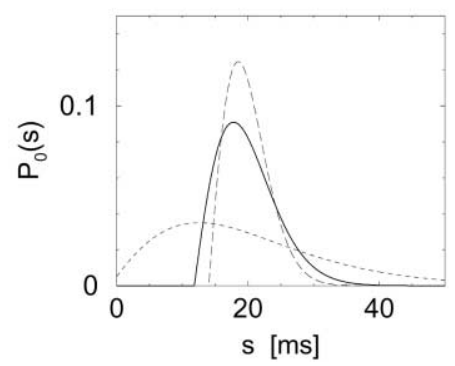

Fig. 8. Reverse correlations (a) and interval distribution (b) for the same neuron as in Fig. 6 , except that the noise level has been changed from $\beta=1$ (solid line) to $\beta=0.1$ (dotted line) or $\beta=2$ (long-dashed line).

potential (PSP) generated by the pulse is

$\epsilon_{0}(s)=\int_{0}^{\infty} \kappa_{0}\left(s^{\prime}\right) \mathscr{I}^{\mathrm{syn}}\left(s-s^{\prime}\right) \mathrm{d} s^{\prime}$.

Let us now suppose that we study a neuron that is spontaneously active at low rate. At $t=t^{\text {in }}$ a presynaptic spike arrives and evokes a PSP. Depending on the internal state of the neuron, the extra PSP may or may not be sufficient to drive the membrane potential across threshold. Intuitively, the cross-correlation function $C^{\text {cross }}\left(t \mid t^{\text {in }}\right)$ describes the probability of generating an output spike given the input. To make our intuition more precise, we take a population approach. Neurons in the population are firing spontaneously at a rate $A_{0}$. At time $t^{\text {in }}$ all neurons receive a presynaptic input spike which generates a modulation $\Delta A(t)$ of the population activity. The change of the neuronal firing rate caused by one presynaptic input spike is $C^{\text {cross }}\left(t \mid t^{\text {in }}\right)=\Delta A(t)$. Using the linearized population equations, we can estimate the cross-correlation

$$
\begin{aligned}
C^{\text {cross }}\left(t \mid t^{\text {in }}\right)=\Delta A(t) \approx & \int_{-\infty}^{t} P_{0}(t-\hat{t}) \Delta A(\hat{t}) \mathrm{d} \hat{t} \\
& +A_{0} \frac{\mathrm{d}}{\mathrm{d} t} \int_{0}^{\infty} \mathscr{L}(x) \epsilon_{0}(t-x) \mathrm{d} x
\end{aligned}
$$

where $P_{0}$ is the interval distribution during spontaneous activity and $A_{0}$ the spontaneous rate. From Eqs. (31) and (32) we conclude that the main peak of the cross-

a)

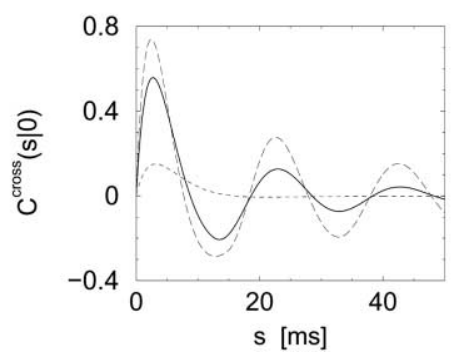

correlation is

$C^{\text {cross }}\left(t^{\text {in }}+s \mid t^{\text {in }}\right)=\left\{\begin{array}{cc}\epsilon_{0}(s) & \text { for high noise } \\ \frac{\mathrm{d}}{\mathrm{d} s} \epsilon_{0}(s) & \text { for low noise. }\end{array}\right.$

Thus, for high noise the cross-correlation is proportional to the PSP while for low noise it is proportional to its derivative (Abeles, 1991; Fetz \& Gustafsson, 1983; Herrmann \& Gerstner, 2001a; Kirkwood \& Sears, 1978; Moore et al., 1970; Poliakov et al., 1997). For intermediate noise levels we calculate the cross-correlation from

$C^{\text {cross }}\left(t^{\text {in }}+s \mid t^{\text {in }}\right)=\int_{0}^{\infty} G\left(s^{\prime}\right) \frac{q}{\tau_{\text {syn }}} \exp \left(-\frac{s-s^{\prime}}{\tau_{\text {syn }}}\right) \mathrm{d} s^{\prime}$.

The result is shown in Fig. 9. For high noise, the time course of the cross-correlation is indeed comparable to that of the PSP; cf Fig. 9b. For low noise, however, the sharp initial rise is followed by a trough and a second peak about one inter-spike interval later. The overall pattern is dominated by oscillations; Fig. 9a.

\section{Discussion}

\subsection{Correlations in spike-time dependent plasticity}

Correlations between pre- and postsynaptic neurons are

b)

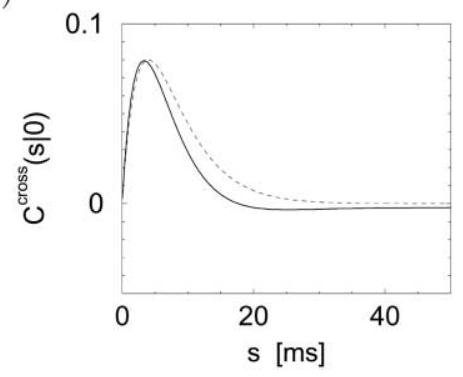

Fig. 9. Cross-correlations. The input consists of a synaptic current pulse at $s=0$ with time constant $\tau_{\mathrm{syn}}=4$ ms. (a) Same neuron model as in Fig. 6, with noise levels $\beta=1$ (solid line), $\beta=0.1$ (dotted line) or $\beta=2$ (long-dashed line). For medium and low noise $(\beta=1$ or 2$)$ the cross-correlations exhibit oscillations with the intrinsic firing period of $20 \mathrm{~ms}$. (b) In the case of high noise ( $\beta=0.05$, solid line) the cross-correlation is similar to the time course of the postsynaptic potential (dotted line). The postsynaptic potential has been scaled to approximately the same amplitude as $C^{\text {cross }}$. 
of eminent importance for synaptic plasticity. In standard Hebbian learning, the weight $w_{i j}$ from a presynaptic neuron $j$ to a postsynaptic neuron $i$ changes if both neurons are active 'at the same time'. Hebb's original formulation is slightly more precise and makes explicit reference to a causal relation between pre- and poststynaptic firing. It states that a change should occur if the 'presynaptic neuron contributes in firing' the postsynaptic one (Hebb, 1949). Thus, learning should occur if there are cross-correlations between pre- and postsynaptic activity.

More generally, we call a plasticity rule Hebbian if

1. it only depends on locally available information such as pre- and postsynaptic spike times and the current weight value $w_{i j}$ and

2 . it depends on the correlations between pre- and postsynaptic firings (i.e. plasticity is not due to pre- or postsynaptic activity alone).

An example of a Hebb-type learning rule is (Gerstner, Kempter, van Hemmen \& Wagner, 1996; Kempter, Gerstner \& van Hemmen, 1999; Kistler, \& van Hemmen, 2000; Roberts \& Bell, 2000; Senn, Tsodyks \& Markram, 2001; Song, Miller \& Abbott, 2000)

$$
\begin{aligned}
\frac{\mathrm{d}}{\mathrm{d} t} w_{i j}(t)= & a_{0}\left(w_{i j}\right)+a_{1}^{\mathrm{in}}\left(w_{i j}\right) S_{j}^{\mathrm{in}}(t)+a_{1}^{\text {out }}\left(w_{i j}\right) S_{i}^{\text {out }}(t) \\
& +a_{2}^{\text {corr }}\left(w_{i j}\right) S_{j}^{\mathrm{in}}(t) \int_{-\infty}^{t} \mathrm{~d} t^{\prime} W\left(t-t^{\prime}\right) S_{i}^{\text {out }}\left(t^{\prime}\right) \\
& +a_{2}^{\text {corr }}\left(w_{i j}\right) S_{i}^{\text {out }}(t) \int_{-\infty}^{t} \mathrm{~d} t^{\prime} W\left(-t+t^{\prime}\right) S_{j}^{\text {in }}\left(t^{\prime}\right),
\end{aligned}
$$

where $S_{j}^{\text {in }}(t)=\sum_{f} \delta\left(t-t_{j}^{(f)}\right)$ is the pre- and $S_{j}^{\text {out }}(t)=$ $\sum_{f} \delta\left(t-t_{j}^{(f)}\right)$ the postsynaptic spike train. The contributions $a_{0}, a_{1}^{\text {in }}, a_{1}^{\text {out }}$ describe the change if there are no spikes at all, presynaptic spikes only, or postsynaptic spikes only. These are called the 'non-Hebbian' terms, since they describe adaptation processes; some or all of these terms may vanish - they are included in Eq. (43) for the sake of generality. All of the above parameters as well as $a_{2}^{\text {corr }}$ may depend on the current weight value $w_{i j}$. The correlations between pre- and postsynaptic spikes are taken care of by the learning window $W\left(t_{j}^{(f)}-t_{i}^{(f)}\right)$ (Gerstner et al., 1996; Gerstner, Ritz \& van Hemmen, 1993). The learning window can have two phases depending on the relative timing of pre- and postsynaptic neurons (Bell, Han, Sugawara \& Grant, 1997; Bi \& Poo, 1998; Debanne, Gähwiler \& Thompson, 1998; Markram, Lübke, Frotscher \& Sakmann, 1997; Zhang, Tao, Holt, Harris \& Poo, 1998). Since such two-phase learning windows are useful to detect temporal variations in neuronal firing patterns, they have been postulated on theoretical grounds (Gerstner et al., 1996).

To see more clearly how correlations enter into the learning equation (43), we assume that learning is slow compared to typical inter-spike intervals. The expected evolution of weights on the slow time scale of learning is ${ }^{2}$

$$
\begin{aligned}
\frac{\mathrm{d}}{\mathrm{d} t} w_{i j}(t)= & a_{0}+a_{1}^{\text {in }}\left\langle S_{j}^{\text {in }}(t)\right\rangle+a_{1}^{\text {out }}\left\langle S_{i}^{\text {out }}(t)\right\rangle \\
& +\int_{-\infty}^{\infty} \mathrm{d} s a_{2}^{\text {corr }} W(s)\left\langle S_{j}^{\text {in }}(t) S_{i}^{\text {out }}(t-s)\right\rangle .
\end{aligned}
$$

The term $\left\langle S_{j}^{\mathrm{in}}(t)\right\rangle$ can be identified with the presynaptic rate $v_{j}^{\text {in }}(t)$ and similarly $\left\langle S_{i}^{\text {out }}(t)\right\rangle$ with the postsynaptic rate $v_{i}^{\text {out }}(t)$. The integral over the learning window is driven by correlations between pre- and postsynaptic spikes. If the presynaptic firing is described by a Poisson process with constant rate, the correlations can be further separated into correlations between pre- and postsynaptic rates and additional spike-spike correlations. The final result is (Kempter et al., 1999)

$$
\begin{aligned}
\frac{\mathrm{d}}{\mathrm{d} t} w_{i j}(t)= & a_{0}+a_{1}^{\text {in }} v_{j}^{\text {in }}+a_{1}^{\text {out }} v_{j}^{\text {out }}+a_{2}^{\text {corr }} v_{j}^{\text {in }} v^{\text {out }} \int_{-\infty}^{\infty} W(s) \mathrm{d} s \\
& +a_{2}^{\text {corr }} v_{j}^{\text {in }} \int_{-\infty}^{\infty} W(-s) C_{i j}^{\text {cross }}(t+s \mid t) \mathrm{d} s .
\end{aligned}
$$

Thus, the cross-correlation $C_{i j}^{\text {cross }}(t+s \mid t)$ enters as expected into the learning equation. Previously, the cross-correlation function $C^{\text {cross }}(t+s \mid t)$ has only been evaluated for a particularly simple stochastically firing neuron model (Kempter et al., 1999). For this simple model it was found that the cross-correlations follow the time course of the postsynaptic potential. The results in this paper allow the analysis of Hebbian plasticity to be extended to more realistic spiking neuron models. As we have seen, the cross-correlation is a linearly filtered version of the postsynaptic potential; the time course of the cross-correlation function depends on the noise level and the mean firing rate of the neuron.

\subsection{Extensions}

In this paper, we calculated reverse and cross-correlations for spiking neuron models. While the framework of the SRM introduced in Section 2 is rather general, we illustrated the results so far only for one specific, somewhat simplified neuron model with piecewise linear escape rate. Extensions could come in several directions. First, we could redo the analysis for more interesting realizations of the SRM (Herrmann \& Gerstner, 2001b; Kistler et al., 1997). Second, if the kernels $\eta$ and $\kappa$ are fitted to real neurons, we could try to explain experimental cross-correlation (Poliakov et al., 1997) or reverse correlation measurements. Third, so far the analysis is restricted to independent neurons. The results should be extended to cross-correlations in connected networks.

\footnotetext{
${ }^{2}$ For the sake of notational simplicity, we suppress the $w_{i j}$ dependence of parameters.
} 


\section{Appendix A. Reverse correlations as a linear filter}

In this appendix, we want to show that the reverse correlation function $C^{\mathrm{rev}}(s)$ is proportional to the filter $G(s)$ of the linear rate model

$v^{\text {out }}(t)=v_{0}^{\text {out }}+\int_{0}^{\infty} G(s) \Delta \mathscr{I}(t-s) \mathrm{d} s$.

Here, $v_{0}^{\text {out }}$ is the mean output rate and $\Delta \mathscr{I}(t)$ is the fluctuating part of the input. Eq. (46) describes the relation between a known (deterministic) input $\Delta \mathscr{I}(t)$ and the rate. We adopt a statistical point of view and assume that the input $\Delta \mathscr{I}(t)$ is drawn from a statistical ensemble of stimuli with mean $\langle\Delta \mathscr{I}(t)\rangle=0$. Angular brackets denote averaging over the input ensemble or, equivalently, over an infinite input sequence. We are interested in the correlation

$$
\begin{aligned}
C_{v \mathscr{I}}(s) & =\lim _{T \rightarrow \infty} \frac{1}{T} \int_{0}^{T} v^{\text {out }}(t+s) \Delta \mathscr{I}(t) \mathrm{d} t \\
& =\left\langle v^{\text {out }}(t+s) \Delta \mathscr{I}(t)\right\rangle
\end{aligned}
$$

between input $\Delta \mathscr{I}$ and activity $\Delta A$. With the linear rate equation (46) we find

$C_{v \mathscr{I}}(s)=\int_{0}^{\infty} G\left(s^{\prime}\right)\left\langle\Delta \mathscr{I}\left(t+s-s^{\prime}\right) \Delta \mathscr{I}(t)\right\rangle \mathrm{d} s^{\prime}$,

where we have used $v_{0}^{\text {out }}\langle\Delta \mathscr{I}(t)\rangle=0$. Thus, the correlation function depends on the filter $G(s)$ as well as on the autocorrelation $\left\langle\Delta \mathscr{I}\left(t^{\prime}\right) \Delta \mathscr{I}(t)\right\rangle$ of the input ensemble.

For the sake of simplicity, we assume that the input consists of white noise, i.e. the input has an autocorrelation

$$
\left\langle\Delta \mathscr{I}\left(t^{\prime}\right) \Delta \mathscr{I}(t)\right\rangle=\sigma^{2} \delta\left(t^{\prime}-t\right) .
$$

In this case, Eq. (48) reduces to

$C_{v \mathscr{I}}(s)=\sigma^{2} G(s)$,

i.e. the correlation function $C_{v \mathscr{I}}$ is proportional to the filter $G(s)$.

In order to relate the correlation function $C_{v \mathscr{I}}$ to the reverse correlation $C^{\text {rev }}$, we think of the rate $v^{\text {out }}$ as the intensity of an inhomogeneous Poisson process that generates spike events $t^{(f)}$. The rate can be estimated if we average over a large number $1 \leq i \leq N$ of repetitions of the experiment (or else over a large number of independent neurons)

$v^{\mathrm{out}}(t)=\frac{1}{N} \sum_{i=1}^{N} \sum_{f} \delta\left(t-t_{i}^{(f)}\right)$.

The correlation function (47) is, therefore,

$$
\begin{aligned}
C_{v \mathscr{I}}(s) & =\lim _{T \rightarrow \infty} \frac{1}{T}\left[\frac{1}{N} \sum_{i=1}^{N} \sum_{f} \Delta \mathscr{I}\left(t_{i}^{(f)}-s\right)\right] \\
& =v_{0}^{\text {out }}\left\langle\mathscr{I}\left(t^{(f)}-s\right)\right\rangle_{t^{(f)}}=v_{0}^{\text {out }} C^{\mathrm{rev}}(s) .
\end{aligned}
$$

For the second equality sign, we have used that the expected number of spike events in an interval of length $T$ is $v_{0}^{\text {out }} T$ where $v_{0}^{\text {out }}$ is the mean rate. The last equality follows from the definition of the reverse correlation function; cf. Eq. (1). The combination of Eqs. (50) and (52) yields

$C^{\mathrm{rev}}(s)=\frac{\sigma^{2}}{v_{0}^{\text {out }}} G(s)$.

Thus, the reverse correlation function is proportional to the linear filter $G$. In Section 4 we calculate the filter $G(s)$ for spiking neurons.

\section{Appendix B1. Integrating the partial differential equation}

In this appendix, we integrate Eq. (21) that describes the dynamics of refractory densities $q(r, t)$. All neurons that have fired together at time $\hat{t}$ form a group that moves along the $r$ axis at constant speed; cf. Fig. 4b. To solve Eq. (21) we turn to a reference frame that moves along with the group. Formally, we replace the variable $r$ by $x=t-r$ and define a new density

$Q(x, t)=q(t-x, t)$ for $x \leq t$

and $Q(x, t)=0$ for $x>t$. The temporal derivative of $Q$ for $x<t$ is

$\frac{\partial}{\partial t} Q(x, t)=\left.\frac{\partial}{\partial r} q(r, t)\right|_{r=t-x} \frac{\mathrm{d} r}{\mathrm{~d} t}+\left.\frac{\partial}{\partial t} q(r, t)\right|_{r=t-x}$

with $\mathrm{d} r / \mathrm{d} t=1$. We insert Eq. (21) on the right-hand-side of Eq. (55). For $x \leq t$, the result is

$\frac{\partial}{\partial t} Q(x, t)=-\rho_{t-x}(t) Q(x, t)+A(t) \delta(t-x)$ for $x \leq t$

and $Q(x, t) \equiv 0$ for $x>t$. Thus, the partial differential equation (21) has been transformed into an ordinary differential equation. The solution of Eq. (56) is (starting the integration at $\left.t_{0}>x\right)$

$Q(x, t)=Q\left(x, t_{0}\right) \exp \left[-\int_{t_{0}}^{t} \rho_{t^{\prime}-x}\left(t^{\prime}\right) \mathrm{d} t\right]$

where $Q\left(x, t_{0}\right)$ is the initial condition of the integration. How can we interpret Eq. (57)? The variable $x=t-r$ is in fact the last firing time $\hat{t}$. Evaluating Eq. (57) in the limit of $t_{0} \rightarrow x$ yields (with $x=\hat{t}$ )

$Q(\hat{t}, t)=A(\hat{t}) \exp \left[-\int_{\hat{t}}^{t} \rho_{t^{\prime}-\hat{t}}\left(t^{\prime}\right) \mathrm{d} t\right]$.

Here, we have used $\lim _{t_{0} \rightarrow \hat{t}} Q\left(\hat{t}, t_{0}\right)=A(\hat{t})$ which follows from the $\delta$ term in Eq. (56). On the other hand, from Eq. (24) we have

$A(t)=\int_{0}^{\infty} \rho_{t-\hat{t}}(t) Q(\hat{t}, t) \mathrm{d} \hat{t}$. 
If we insert Eq. (58) into Eq. (59), we find

$A(t)=\int_{-\infty}^{t} \rho_{t-\hat{t}}(t) \exp \left[-\int_{\hat{t}}^{t} \rho_{t^{\prime}-\hat{t}}\left(t^{\prime}\right) \mathrm{d} t^{\prime}\right] A(\hat{t}) \mathrm{d} \hat{t}$

which is the population equation (16) for escape noise. If we insert Eq. (58) into the normalization condition for the $Q$-variable, $1=\int_{-\infty}^{t} Q(\hat{t}, t) \mathrm{d} t$, we arrive at a normalization equation for the activity:

$1=\int_{-\infty}^{t} \exp \left(-\int_{\hat{t}}^{t} \rho_{t^{\prime}-\hat{t}}\left(t^{\prime}\right) \mathrm{d} t^{\prime}\right) A(\hat{t}) \mathrm{d} \hat{t}$.

Eq. (61) can be used as a starting point for a theory of population dynamics (Gerstner, 2000). In fact, Eq. (6) can be derived from Eq. (61) by taking the temporal derivative.

\section{Appendix B2. From membrane potential densities to refractory densities}

In this appendix, we study a population of integrate-andfire neurons with escape noise. We want to show that we can use the membrane potential trajectory of the integrate-andfire model

$u(t)=\eta_{0} \exp \left(-\frac{t-\hat{t}}{\tau}\right)+\int_{\hat{t}}^{t} \exp \left(-\frac{t-t^{\prime}}{\tau}\right) \mathscr{I}\left(t^{\prime}\right) \mathrm{d} t^{\prime}$

to define a transformation from voltage to refractory variables: $u \rightarrow r$ with $r=t-\hat{t}$. It turns out that the final equations are even simpler if we take $\hat{t}$ instead of $r$ as our new variable-and we, therefore, consider the transformation $u \rightarrow \hat{t}$.

Before we start, we calculate the derivatives of Eq. (62). The derivative with respect to $t$ yields $\partial u / \partial t=[-u / \tau]+$ $\mathscr{I}(t)$ as expected for integrate-and-fire neurons. The derivative with respect to $\hat{t}$ is

$$
\frac{\partial u}{\partial \hat{t}}=\frac{\eta_{0}-R \mathscr{I}(t)}{\tau} \exp \left(-\frac{t-\hat{t}}{\tau}\right)=F(t, \hat{t})\left\{\frac{\eta_{0}}{\tau}\right\}-\mathscr{I}(\hat{t}) \exp
$$

where the function $F$ is defined by Eq. (63).

The densities in the variable $\hat{t}$ are denoted as $Q(\hat{t}, t)$. Since the density $Q$ is normalized to one, the coordinate transformation from $p(u, t)$ to $Q(\hat{t}, t)$ must respect $Q(\hat{t}, t) \mathrm{d} \hat{t}=$ $p(u, t) \mathrm{d} u$. This yields

$Q(\hat{t}, t)=p(u, t) F(t, \hat{t})$

where $u$ is taken as a function of $t$ and $\hat{t}$; cf. Eq. (62). We now want to show that the differential equation for the density $Q(\hat{t}, t)$ that we derived in Eq. (56)

$$
\frac{\partial}{\partial t} Q(\hat{t}, t)=-\rho_{t-\hat{t}}(t) Q(\hat{t}, t) \text { for } \hat{t}<t
$$

is equivalent to the continuity equation (17) for the membrane potential densities. If we insert Eq. (64) into
Eq. (65) we find

$\frac{\partial p}{\partial u} \frac{\partial u}{\partial t} F+\frac{\partial p}{\partial t} F+p \frac{\partial F}{\partial t}=-\rho p F$.

For the integrate-and-fire neuron, we have $\partial F / \partial t=-F / \tau$. Furthermore, for $R \mathscr{I}(t)>\eta_{0}$ we have $F \neq 0$. Thus, we can divide Eq. (66) by $F$ and rewrite Eq. (66) in the form

$$
\frac{\partial p(u, t)}{\partial t}=-\frac{\partial}{\partial u}[\dot{u} p(u, t)]-f(u-\vartheta) p(u, t) \text { for } u>u_{r} .
$$

\section{References}

Abbott, L. F., \& van Vreeswijk, C. (1993). Asynchronous states in a network of pulse-coupled oscillators. Phys. Rev. E, 48, 1483-1490.

Abeles, M. (1991). Corticonics, Cambridge: Cambridge University Press.

Amit, D. J., \& Brunel, N. (1997). A model of spontaneous activity and local delay activity during delay periods in the cerebral cortex. Cerebral Cortex, 7, 237-252.

Bell, C., Han, V., Sugawara, Y., \& Grant, K. (1997). Synaptic plasticity in a cerebellum-like structure depends on temporal order. Nature, 387, 278281

Berry, M., \& Meister, M. (1998). Refractoriness and neural precision. $J$. Neurosci., 18, 2200-2211.

Bethge, M., Pawelzik, K., Rothenstein, R., \& Tsodyks, M. (2001). Noise as a signal for neuronal populations (submitted).

Bi, G., \& Poo, M. (1998). Synaptic modifications in cultured hippocampal neurons: dependence on spike timing, synaptic strength, and postsynaptic cell type. J. Neurosci., 18, 10464-10472.

Bialek, W., Rieke, F., de Ruyter van Steveninck, R. R., \& Warland, D. (1991). Reading a neural code. Science, 252, 1854-1857.

Brunel, N., \& Hakim, V. (1999). Fast global oscillations in networks on integrate-and-fire neurons with low firing rates. Neural Computation, $11,1621-1671$

Brunel, N., Chance, F., Fourcaud, N., \& Abbott, L. (2001). Effects of synaptic noise and filtering on the frequency response of spiking neurons. Physical Review Letters, 86, 2186-2189.

Cox, D. R. (1962). Renewal theory, London: Methuen.

Debanne, D., Gähwiler, B., \& Thompson, S. (1998). Long-term synaptic plasticity between pairs of individual CA3 pyramidal cells in rat hippocampal slice cultures. J. Physiol., 507, 237-247.

de Boer, E., \& Kuyper, P. (1968). Triggered correlation. IEEE Trans. Biomedical Engineering, 15, 169-179.

de Ruyter van Steveninck, R. R., Lowen, G. D., Strong, S. P., Koberle, R., \& Bialek, W. (1997). Reproducibility and variability in neural spike trains. Science, 275, 1805.

Destexhe, A., \& Pare, D. (1999). Impact of network activity on the integrative properties of neocortical pyramidal neurons in vivo. Journal of Neurophysiology, 81, 1531-1547.

Fetz, E. E., \& Gustafsson, B. (1983). Relation between shapes of postsynaptic potentials and changes in firing probability of cat motoneurons. J. Physiol., 341, 387-410.

Fusi, S., \& Matteo, M. (1999). Collective behaviour of networks with linear (VLSI) integrate and fire neurons. Neural Computation, 11, 633-652.

Gerstner, W. (1995). Time structure of the activity in neural network models. Phys. Rev. E, 51 (1), 738-758.

Gerstner, W. (2000). Population dynamics of spiking neurons: fast transients, asynchronous states and locking. Neural Computation, 12, 43-89.

Gerstner, W., \& van Hemmen, J. L. (1992). Associative memory in a network of 'spiking' neurons. Network, 3, 139-164.

Gerstner, W., \& van Hemmen, J. L. (1994). Coding and information processing in neural networks. In E. Domany, J. L. van Hemmen \& 
K. Schulten, Models of neural networks II (pp. 1-93). New York: Springer-Verlag.

Gerstner, W., Kempter, R., van Hemmen, J. L., \& Wagner, H. (1996). A neuronal learning rule for sub-millisecond temporal coding. Nature, $386,76-78$.

Gerstner, W., Ritz, R., \& van Hemmen, J. L. (1993). Why spikes? Hebbian learning and retrieval of time-resolved excitation patterns. Biol. Cybern., 69, 503-515.

Hebb, D. O. (1949). The organization of behavior, New York: Wiley.

Herrmann, A., \& Gerstner, W. (1999). Understanding the psth response to synaptic input. In ICANN '99 Artificial Neural Networks (vol. 270, pp. 1012-1017). IEE Conference Publication.

Herrmann, A., \& Gerstner, W. (2001). Noise and the psth response to current transients. J. Computational Neuroscience (in press).

Herrmann, A., \& Gerstner, W. (2001). Noise and the psth reponse to current transients. J. Computational Neuroscience (in press).

Kempter, R., Gerstner, W., \& van Hemmen, J. L. (1999). Hebbian learning and spiking neurons. Phys. Rev. E, 59, 4498-4514.

Kirkwood, P., \& Sears, P. (1978). The synaptic connexions to intercostal motoneurones as revealed by the average common excitation potential. J. Physiol., 275, 103-134.

Kistler, W. M., \& van Hemmen, J. L. (2000). Modeling synaptic plasticity in conjunction with the timing of pre- and postsynaptic potentials. Neural Comput., 12, 385-405.

Kistler, W. M., Gerstner, W., \& van Hemmen, J. L. (1997). Reduction of Hodgkin-Huxley equations to a single-variable threshold model. Neural Comput., 9, 1015-1045.

Kjaer, T. W., Hertz, J. A., \& Richmond, B. J. (1994). Decoding cortical neuronal signals: network models, information estimation and spatial tuning. J. Computational Neuroscience, 1, 109-139.

Knight, B. W. (1972a). Dynamics of encoding in a population of neurons. $J$. Gen. Physiology, 59, 734-766.

Knight, B. W. (1972b). The relationship between the firing rate of a single neuron and the level of activity in a population of neurons. J. Gen. Physiology, 59, 767-778.

Knox, C. K. (1974). Cross-correlation functions for a neuronal model. Biophys. J., 14, 567-582.

König, P., Engel, A. K., \& Singer, W. (1996). Integrator or coincidence detector? The role of the cortical neuron revisited. TINS, 19 (4), 130137.

Maas, W., \& Nathschläger, T. (2000). A model for fast analog computation based on unreliable synapses. Neural Computation, 12, 1679-1704.

Markram, H., Lübke, J., Frotscher, M., \& Sakmann, B. (1997). Regulation of synaptic efficacy by coincidence of postsynaptic ap and epsp. Science, 27, 213-215.
Moore, G., Segundo, J., Perkel, D., \& Levitan, H. (1970). Statistical signs of synaptic interaction in neurons. Biophys. Journal, 10, 876-900.

Nykamp, D., \& Tranchina, D. (2000). A population density approach that facilitates large-scale modeling of neural networks: analysis and application to orientation tuning. J. Computational Neuroscience, 8 , $19-50$.

Omurtag, A., Knight, B., \& Sirovich, L. (2000). On the simulation of a large population of neurons. J. Computational Neuroscience, 8, 51-63.

Optican, L. M., \& Richmond, B. J. (1987). Temporal encoding of twodimensional patterns by single units in primate inferior temporal cortex. 3. Information theoretic analysis. J. Neurophysiol., 57, 162-178.

Perkel, D. H., Gerstein, G. L., \& Moore, G. P. (1967). Neuronal spike trains and stochastic point processes. I. The single spike train. Biophys. J., 7, $391-418$

Plesser, H. E., \& Gerstner, W. (2000). Noise in integrate-and-fire models: from stochastic input to escape rates. Neural Computation, 12, 367384.

Poliakov, A. V., Powers, R. K., \& Binder, M. C. (1997). Functional identification of input-output transforms of motoneurons in cat. J. Physiology, 504, 401-424.

Riccardi, L. M. (1977). Diffusion processes and related topics in biology, Berlin: Springer-Verlag.

Rieke, F., Warland, D., de Ruyter van Steveninck, R., \& Bialek, W. (1996). Spikes-exploring the neural code, Cambridge, MA: MIT Press.

Roberts, P., \& Bell, C. (2000). Computational consequence of temporally asymmetric learning rules: sensory image cancellation. J. Computational Neuroscience, 9, 67-83.

Roddey, J., Girish, B., \& Miller, J. (2000). Assessing the performance of neural encoding models in the presence of noise. J. Computational Neuroscience, 8, 95-112.

Senn, W., Tsodyks, M., \& Markram, H. (2001). An algorithm for modifying neuro-transmitter release probability based on pre- and postsynaptic spike timing. Neural Computation, 13, 35-67.

Softky, W. R. (1995). Simple codes versus efficient codes. Current Opinion in Neurobiology, 5, 239-247.

Song, S., Miller, K., \& Abbott, L. (2000). Competitive Hebbian learning through spike-time-dependent synaptic plasticity. Nature Neuroscience, 3, 919-926.

Tovee, M. J., Rolls, E. T., Treves, A., \& Belles, R. P. (1993). Information encloding and the responses of single neurons in the primate visual cortex. J. Neurophysiol., 70, 640-654.

Zhang, L., Tao, H., Holt, C., Harris, W. A., \& Poo, M. -M. (1998). A critical window for cooperation and competition among developing retinotectal synapses. Nature, 395, 37-40. 